\title{
Multiple lidocaine infusions for relief of neuropathic pain: systematic review and meta-analysis
}

\author{
Antônio Silvinato ${ }^{1}$ \\ (D) Idevaldo Floriano ${ }^{1}$ \\ Wanderley Marques Bernardo²
}

1. Programa Diretrizes $D a A M B$, São Paulo, $S P$, Brasil 2. Coordenador Do Programa Diretrizes Da AMB, São Paulo, SP, Brasil

Email: Contato: wmbernardo@usp.br

http://dx.doi.org/10.1590/1806-9282.66.7.889

QUESTION: The clinical question is: What is the impact of therapy with multiple infusions of lidocaine on outcomes of pain relief for up to four weeks and adverse events in the treatment of patients with neuropathic pain, compared with a placebo?'

Answer: In patients with neuropathic pain, infused lidocaine once a week, for 4 weeks, compared with a placebo (saline solution $0.9 \%$ ) showed no difference in pain reduction in up to 4 weeks. Moderate quality of evidence. Intravenous lidocaine increases the risk of adverse events (any) in 25\% (95\% CI 18 to $31 \%$ ) in comparison with a placebo (saline solution $0.9 \%$ ), and it is necessary to treat 4 patients for one to present an adverse event (95\% CI 3 to 6). Low quality of evidence. Dizziness, drowsiness, perioral paresthesia, nausea, headache, dysarthria, dry mouth, metallic taste are some of the most common side effects.

\section{REFERENCE}

1. Silvinato A, Floriano I, Bernardo WM - Multiple lidocaine infusions for relief of neuropathic pain: systematic review and meta-analysis - Rev Assoc Med Bras 2020; 66(5): 583-588. 